\title{
Cue and Reward Evoked Dopamine Activity Is Necessary for Maintaining Learned Pavlovian Associations
}

\author{
Ruud van Zessen, ${ }^{1}$ Jacques P. Flores-Dourojeanni, ${ }^{1}$ Timon Eekel, ${ }^{1}$ Siem van den Reijen, ${ }^{1}$ Bart Lodder, ${ }^{1}$ \\ ${ }^{\circledR}$ Azar Omrani, ${ }^{1}$ Marten P. Smidt, ${ }^{2}{ }^{\circledR}$ Geert M.J. Ramakers, ${ }^{1}$ Geoffrey van der Plasse, ${ }^{1}{ }^{-}$Garret D. Stuber, ${ }^{3}$ and \\ (ㅈoger A.H. Adan ${ }^{1,4}$ \\ ${ }^{1}$ Department of Translational Neuroscience, Brain Center Rudolf Magnus, University Medical Center Utrecht, 3584 CG Utrecht, The Netherlands, \\ ${ }^{2}$ Molecular Neuroscience, Swammerdam Institute for Life Sciences, University of Amsterdam, 1090 GE Amsterdam, The Netherlands, ${ }^{3}$ Center for \\ the Neurobiology of Addiction, Pain, and Emotion, Department of Anesthesiology and Pain Medicine, Department of Pharmacology, University of \\ Washington, Seattle, Washington 98195, and ${ }^{4}$ Institute of Neuroscience and Physiology, The Sahlgrenska Academy at the University of Gothenburg, \\ 40530 Gothenburg, Sweden
}

Associating natural rewards with predictive environmental cues is crucial for survival. Dopamine (DA) neurons of the ventral tegmental area (VTA) are thought to play a crucial role in this process by encoding reward prediction errors (RPEs) that have been hypothesized to play a role in associative learning. However, it is unclear whether this signal is still necessary after animals have acquired a cue-reward association. In order to investigate this, we trained mice to learn a Pavlovian cue-reward association. After learning, mice show robust anticipatory and consummatory licking behavior. As expected, calcium activity of VTA DA neurons goes up for cue presentation as well as reward delivery. Optogenetic inhibition during the moment of reward delivery disrupts learned behavior, even in the continued presence of reward. This effect is more pronounced over trials and persists on the next training day. Moreover, outside of the task licking behavior and locomotion are unaffected. Similarly to inhibitions during the reward period, we find that inhibiting cue-induced dopamine (DA) signals robustly decreases learned licking behavior, indicating that cue-related DA signals are a potent driver for learned behavior. Overall, we show that inhibition of either of these DA signals directly impairs the expression of learned associative behavior. Thus, continued DA signaling in a learned state is necessary for consolidating Pavlovian associations.

Key words: dopamine; optogenetics; Pavlovian conditioning; reward prediction error; ventral tegmental area

Significance Statement

Dopamine (DA) neurons of the ventral tegmental area (VTA) have long been suggested to be necessary for animals to associate environmental cues with rewards that they predict. Here, we use time-locked optogenetic inhibition of these neurons to show that the activity of these neurons is directly necessary for performance on a Pavlovian conditioning task, without affecting locomotor per se These findings provide further support for the direct importance of second-by-second DA neuron activity in associative learning.

Received Oct. 26, 2020; revised Feb. 21, 2021; accepted Mar. 16, 2021

Author contributions: R.v.Z., G.v.d.P., G.M.J.R., G.D.S., and R.A.H.A. designed research; R.v.Z., J.P.F.-D., T.E., S.v.d.R.,

B.L., and A.O. performed research; M.P.S. contributed unpublished reagents/analytic tools; R.v.Z., J.P.F.-D., T.E., S.v.d.R.,

B.L., and A.O. analyzed data; R.v.Z., G.v.d.P., G.M.J.R., and R.A.H.A. wrote the paper.

This work was supported by The Netherlands Organization for Scientific Research NWO/ALW Veni Grant \#863.13.018 and by the European Union's Seventh Framework Program for Research, Technological Development and Demonstration Grant No. 266408 (Full4Health).

R. van Zessen's present address: Department of Basic Neurosciences, Faculty of Medicine, University of Geneva, 1211 Geneva, Switzerland.

The authors declare no competing financial interests.

Correspondence should be addressed to Roger A.H. Adan at r.a.h.adan@umcutrecht.nl.

https://doi.org/10.1523/JNEUROSCI.2744-20.2021

Copyright $\odot 2021$ the authors

\section{Introduction}

Dopamine (DA) neurons projecting from the ventral tegmental area (VTA) of the midbrain to the striatum are known for their involvement in motivational processes and the formation of stimulus-reward associations (Wise, 2004; Fields et al., 2007; Salamone and Correa, 2012). As such, increased DA activity drives reward seeking behavior, both for natural rewards like food (Roitman et al., 2004; Adamantidis et al., 2011), as well as drugs of abuse (Phillips et al., 2003; Pascoli et al., 2015). Disturbances in the function of these neurons and downstream target areas like the nucleus accumbens (NAc) are suggested to contribute to multiple psychiatric disorders, including addiction, mood disorders, schizophrenia, and attention-deficit hyperactivity disorder (Castellanos and Tannock, 2002; Nestler and 
Carlezon, 2006; Howes and Kapur, 2009). However, fundamental understanding of the necessity of DA neuronal activity for associative behavior remains limited.

Midbrain DA neurons show endogenous burst firing activity when an animal encounters an unexpected reward, which transfers to predictive cues as an animal learns to associate environmental stimuli with the availability of a reward (Schultz et al., 1997; Pan et al., 2005; Day et al., 2007). These signals have been suggested to encode a reward prediction error (RPE; Schultz et al., 1997). Others have suggested that DA signaling mediates appetitive responses to reward-predicting stimuli, through attributing incentive salience (Berridge, 2007), mediating behavioral activation (Robbins and Everitt, 1992, 2007), or mediating flexible approach behavior (Nicola, 2010).

A fundamental question that arises is whether these signals are necessary for the development and expression of associative behavior. Pharmacological, genetic, and lesion studies have implicated NAc DA in the acquisition (Di Ciano et al., 2001; Parkinson et al., 2002; Zweifel et al., 2009), as well as the expression of associative conditioned behavior (Parkinson et al., 2002; Nicola et al., 2005). However, these manipulations cause longterm deficiencies in DA signaling, while behaviorally relevant DA signals occur on subsecond timescales (Schultz et al., 1997).

Optogenetic transient inhibition of VTA DA neurons can disrupt model-based associative learning (Sharpe et al., 2017) and facilitate extinction learning (Chang et al., 2016). Interestingly, these effects were observed on reward-related learning in the session following DA manipulations, but not directly on task execution, implying a confined delayed role of DA in learning. In contrast a more recent study finds that DA inhibition during the reward period in Pavlovian conditioned head-fixed mice directly affects both consummatory and anticipatory licking behavior. Surprisingly this study does not find any longer lasting effects of this inhibition (Lee et al., 2020). Moreover, this study also finds no effects of VTA DA inhibition during the cue period in trained animals. It remains to be seen whether these manipulations have the same effect on freely-moving animals, and whether differences in task-setup can explain this apparent difference in delayed versus immediate effects of DA inhibition.

In earlier work, we established that activation of VTA GABA neurons during a cue-reward seeking conditioning task, specifically decreased reward consummatory behavior (van Zessen et al., 2012). While we show that VTA GABA neurons monosynaptically inhibit VTA DA neurons, these effects could also partially be mediated through VTA GABA projections to other areas (Brown et al., 2012), co-transmission of other neurotransmitters (Stuber et al., 2010; Root et al., 2014), or biased connectivity between VTA GABA neurons and subsets of VTA DA neurons (Yang et al., 2018). Here, we establish the effect of direct inhibition of VTA DA neurons during expression of Pavlovian conditioned behavior. As VTA DA neurons are endogenously active during cue and reward presentation (Cohen et al., 2012), we chose to inhibit these neurons during these time periods. We find that optogenetic inhibition of VTA DA activity during reward presentation acutely attenuates reward-seeking behavior, in line with recent work (Lee et al., 2020). However, we also find this effect progresses over time, and is still present on the next day. These inhibitions of VTA DA neurons are insufficient to modulate licking or locomotor performance outside of the cue-reward conditioning task, ruling out pure motoric or aversive effects. Moreover, we find that inhibition of VTA DA neurons during the cue period also causes pronounced decreases in behavioral performances on the
Pavlovian conditioning task. Overall, these data suggest that a time-locked inhibition of VTA DA neurons causes a decrease in the potency of the cue and reward's ability to generate a behavioral reinforcement signal, directly impacting current and future behavioral responding.

\section{Materials and Methods}

Animals and housing

Experiments were approved by the Central Commission Animal Experiments (Centrale Commissie Dierproeven; CCD) or the Animal Experimentation Committee (Dier Experimentele Commissie, DEC) of the University Utrecht and conducted in agreement with Dutch Law (Herziene Wet op Dierproeven, Art 10.a.2, 2014) and European regulations (Guidelines 86/609/EEC and 2010/63/EU).

Adult male Pitx3-CRE heterozygote animals on a C57BL/6J background were kept on a reversed (lights on 7 A.M.) 12/12 h light/dark cycle, and ran for experiments during the night (active) cycle. Following recovery from surgery they were solitary housed and food restricted to $90 \%$ of their ad libitum weight for the duration of the experiments.

\section{Surgery}

Mice were anesthetized with intraperitoneally administered ketamine $(75 \mathrm{mg} / \mathrm{kg})$ and medetomidine $(1 \mathrm{mg} / \mathrm{kg})$. They were then placed in a stereotactic frame (Kopf Instruments), and microinjections were performed in the VTA $\left(-3.2 \mathrm{AP},-1.5 \mathrm{ML},-4.8 \mathrm{DV}, 15^{\circ}\right.$ angle). For optogenetic manipulations a volume of 300-nl CRE-inducible halorhodopsin (NPHR), or yellow fluorescent protein (YFP) containing adeno associated virus (AAV2.5-EF1a-DIO-eNPHR3.0-EYFP or AAV2.5-EF1a-DIO-EYFP, respectively; UNC Vectore Core) was bilaterally injected at a rate of $0.1 \mu \mathrm{l} / \mathrm{min}$ per side, followed by an additional $10 \mathrm{~min}$ period before injection needles were retracted. For fiber photometry recordings, 300-500 $\mathrm{nl}$ of CRE-inducible GCaMP6s (AAV2.5-FLEX-hSyn-GCaMP6s, UPENN Vector Core) was injected unilaterally into the VTA in a similar manner. Afterwards, chronic implantable optic fibers were placed above the injections sites, which were attached to the skull using dental cement (GC Fuji Plus) and skull screws. Animals were treated with carprofen $(5 \mathrm{mg} / \mathrm{kg})$, atipamezole $(2.5 \mathrm{mg} / \mathrm{kg})$, and saline for rehydration following surgery. They were given carprofen for the subsequent $2 \mathrm{~d}$ and then allowed to recover for at least one week before starting training.

\section{Optogenetic manipulation}

Optogenetic implantable chronic fibers and optical patch cables were constructed as described previously (Sparta et al., 2011). Briefly, chronic fibers consisted of a $200-\mu \mathrm{m}$ core multimode fiber (FT200UMT, Thorlabs) were attached to ceramic zirconia ferrules (MM-FER2007C2300, Precision Fiber Products) and cut to a length of $6 \mathrm{~mm}$. Patch cables consisted of a $50-\mu \mathrm{m}$ core multimode fiber (FG050UGA, Thorlabs) threaded through furcation tubing (FF9-250-BLA-100, Precision Fiber Products), and terminated in a ceramic zirconia ferrule (MMFER2007C1270, Precision Fiber Products) on one end and a multimode FC/PC connector (MM-CON2004-1270-14-BLK, Precision Fiber Products) on the other end. These were connected to a fiber optic rotary joint (FRJ_1x2i_FC-2FC_0.22, Doric Lenses). A second patch cable connected the rotary joint with a diode-pumped solid-state laser (MGL-III-532, CNI Lasers). Unless otherwise specified, lasers were calibrated to deliver at least $5 \mathrm{~mW}$ onto the VTA. After surgery, but before any behavioral assay, animals were tethered to optogenetic patch cables in their homecage to habituate them to the handling procedure.

Fiber photometry recording

In order to perform fiber photometry recordings excitation LED blue and purple light (LEDC1-B_FC and LEDC1405_FC, Doric Lenses) were frequency and phase locked at 208 and $531 \mathrm{~Hz}$ using a lock-in modulator (FPC, Doric) passed through an optical minicube [FMCS_AE(405)_AF (420-450)_E1(460-490)_F(500-550)_S, Doric] before being coupled to a patch cable connecting to implanted animal (MFP_400/460/900- 
0.48_3m_FCM-MF2.5, Doric). Green emission light then traveled through the same fiber optics, through the dichroic mirror and emission filter in the minicube, and was focused onto a silicon based photoreceiver (\#2151 Photoreceiver, Newport Corporation). The signal was then preamplified $(2 \times 1010 \mathrm{~V} / \mathrm{A}$ gain $)$ at the photoreceiver, digitized and saved at the fiber photometry console (FPC, Doric). Additional TTL signals from behavioral events were simultaneously recorded. Data were then processed using Python and MATLAB, where the 405 signal was used as an isobestic control and subtracted from the signal (Lerner et al., 2015). Data were then aligned to cue onset period and Z-scored.

\section{Cue-reward conditioning}

Animals were trained for $5 \mathrm{~d}$ per week on a Pavlovian conditioning task. On the first day, they were habituated to the operant cage, with ad libitum $20 \%$ sucrose present in the reward receptacle (CT-ENV-303LP-DP3, Med Associates). The operant chamber (ENV-307W, Med Associates) contained an electrical circuit lickometer (ENV-250, Med Associates), as well as a houselight and tone generator (Resp. ENV-315W and ENV224AM, Med Associates). Typical training consisted of a 1-h training sessions, in which 40 identical trials were presented with a pseudo-random inter-trial interval of 60-120 s. Trials consisted of an auditory and visual cue that were presented for $5 \mathrm{~s}$, followed immediately by delivery of $8 \mu \mathrm{l}$ of $20 \%$ sucrose into the reward receptacle by a pump (PHM$100 \mathrm{~A}$, Med Associates). Animals were tethered to optogenetic cables during training, and were extensively trained with "mock" stimulation (where laser light is blocked from entering the brain) to habituate them to the light delivery. Lick and cue presentation timestamps were registered and used for analyses. Licks that were made after cue onset but before reward delivery were counted as "anticipatory" licks, while licks made after reward delivery were counted as "consummatory" licks. A small fraction of animals $(\sim 1$ in 20$)$ did not learn the task at all, as they did not show many anticipatory or consummatory licks, and typically did not drink the presented sucrose reward. These animals have been excluded from the study.

\section{Locomotion assay}

Following completion of Pavlovian conditioning experiments, a subset of animals was used to assess locomotion responses. Animals were tethered to an optical cable, placed in $32 \times 25 \mathrm{~cm}$ square arena, and allowed to move freely for $30 \mathrm{~min}$. Green light was delivered for $5 \mathrm{~s}$ every $90 \mathrm{~s}$ for the duration of the experiment (same frequency as during cue-reward seeking). Video tracks were analyzed using Ethovision (Noldus Information Technology), distance moved over time was extracted, and subsequent analyses were done in MATLAB (MathWorks) and Excel (Microsoft).

\section{Free consumption assay}

In order assess the possible effects of DA inhibition of ongoing licking behavior, a separate batch of animals were trained to consume ad libitum sucrose for $30 \mathrm{~min}$ in the same operant chamber as described above. Once total lick responding was stable ( $<15 \%$ change over $2 \mathrm{~d}$ ) animals optogenetic manipulations were started. In order to stimulate after lick bout initiation, the laser was triggered after three licks within $0.5 \mathrm{~s}$, followed by a 20 -s minimum interstimulation interval. These criteria ensured a similar stimulation frequency $(21.3 \pm 5.9$ per 30 min; mean \pm SEM) compared with the cue-reward conditioning task (40 per $60 \mathrm{~min}$ ). Lick and laser onset timestamps were used for analysis.

\section{Ex vivo electrophysiology}

Virus-injected animals were anesthetized by intraperitoneal injection of sodium pentobarbital $(200 \mathrm{mg} / \mathrm{kg})$. Horizontal midbrain slices $(250 \mu \mathrm{m})$ were prepared using a vibratome (Leica VT1200S, Leica Microsystems) after transcardiac perfusion with ice-cold modified artificial CSF (ACSF) containing the following: $75 \mathrm{~mm}$ sucrose, $87 \mathrm{~mm} \mathrm{NaCl}, 2.5 \mathrm{~mm} \mathrm{KCl}, 25$ $\mathrm{mm} \mathrm{NaH} \mathrm{PO}_{4}, 7 \mathrm{~mm} \mathrm{MgCl}_{2}, 0.5 \mathrm{~mm} \mathrm{CaCl}_{2}, 25 \mathrm{~mm} \mathrm{NaHCO}, 1.25 \mathrm{~mm}$ glucose, and $5 \mathrm{~mm}$ ascorbate (oxygenated with $95 \% \mathrm{O}_{2}$ and $5 \% \mathrm{CO}_{2}, \mathrm{pH}$ 7.3-7.4). Slices were then transferred into a holding chamber containing standard ACSF containing the following: $126 \mathrm{~mm} \mathrm{NaCl}, 3 \mathrm{~mm} \mathrm{KCl}, 2 \mathrm{~mm}$ $\mathrm{MgSO}_{4}, 2 \mathrm{~mm} \mathrm{CaCl}, 10 \mathrm{~mm}$ glucose, $1.25 \mathrm{~mm} \mathrm{NaH}_{2} \mathrm{PO}_{4}$, and $26 \mathrm{~mm}$ $\mathrm{NaHCO}_{3}$ (oxygenated with $95 \% \mathrm{O}_{2}$ and $5 \% \mathrm{CO}_{2}, \mathrm{pH} 7.4$ ) at $34^{\circ} \mathrm{C}$ for
$30 \mathrm{~min}$ and allowed to recover for at least $30 \mathrm{~min}$ at room temperature. Slices were then placed in a recording chamber, where they were continuously perfused with oxygenated ACSF at $30-32^{\circ} \mathrm{C}$. Cells were visualized with an Olympus BX61W1 microscope equipped with infrared differential interference contrast optics and VTA DA neurons expressing eNpHR-eYFP identified by fluorescence microscopy. Patch electrodes were pulled from borosilicate glass capillaries and had a resistance of 3-5 $\mathrm{M} \Omega$ when filled with intracellular solution contained the following: $140 \mathrm{~mm}$ K-gluconate, $1 \mathrm{~mm} \mathrm{KCl}, 10 \mathrm{~mm}$ HEPES, $0.5 \mathrm{~mm}$ EGTA, $4 \mathrm{~mm}$ MgATP, $0.4 \mathrm{~mm} \mathrm{Na}_{2} \mathrm{GTP}$, and $4 \mathrm{~mm}$ phosphocreatine ( $\mathrm{pH} 7.3$ with $\mathrm{KOH})$. Whole-cell patch-clamp recordings were made using EPC10 patch-clamp amplifier and PatchMaster v2x73 software (HEKA Electronics). Signals were low-pass filtered at $3 \mathrm{kHz}$ and digitized at $10 \mathrm{kHz}$. Series resistance was constantly monitored and cells were excluded from analysis if the resistance changed by $>20 \%$. Photostimulation was performed by a TTL controlled 532-nm diodepumped solid-state laser (MGL-III-532, CNI Lasers) delivered via an optic fiber (50- $\mu \mathrm{m}$ diameter, ThorLabs) positioned close to the surface of the slice. Light intensity was adjusted to obtain a power of $1 \mathrm{~mW}$ (measured at the fiber tip). Data were analyzed with Clampfit 10 (Molecular Devices) software.

\section{Immunohistochemistry}

Animals were anesthetized with sodium pentobarbital $(200 \mathrm{mg} / \mathrm{kg})$, and then transcardially perfused with PBS followed by $4 \%$ paraformaldehyde in PBS (PFA). Brains were kept on PFA for $24 \mathrm{~h}$ at $4^{\circ} \mathrm{C}$ and then transferred to $30 \%$ sucrose for at least $48 \mathrm{~h}$ at $4^{\circ} \mathrm{C}$. They were then sectioned to $40-\mu \mathrm{m}$ slices using a cryostat and stored in PBS containing $0.01 \%$ sodium azide. They were blocked for $2 \mathrm{~h}$ in PBS containing $10 \%$ goat serum and $1 \%$ Triton X-100. Primary antibody (chicken anti-GFP 1:500, rabbit anti-TH 1:750) in PBS containing $10 \%$ goat serum was left on $\mathrm{O} / \mathrm{N}$ at $4^{\circ} \mathrm{C}$. Slices were then washed for four to five times for $30 \mathrm{~min}$, and incubated with secondary antibody (goat anti-chicken Alexa Fluor 488 1:500, goat anti-rabbit Alexa Fluor 594 1:500) for $2 \mathrm{~h}$ at RT. They were then washed an additional four to five times, transferred onto slides and covered using a coverslip. Images (single plane or z-stacks) were collected on an epifluorescent microscope (Axio Scope A1, Zeiss) or confocal microscope (FV1000, Olympus). Animals that did not have fibers in the VTA or did not have any viral expression were excluded from the analysis.

\section{Analyses}

Animals in which implanted chronic optic fibers detached during the experiments, as well as animals that showed unilateral expression of NPHR were excluded from all analyses. Behavioral data were analyzed using Excel, Neuroexplorer (NEXTechnologies), and MATLAB. To assess licking behavior on the cue-reward seeking task and the free consumption task, perievent histograms were constructed that compare lick activity around cue onset. Data from stimulation day was compared with prestimulation mock day. Subsequent statistical analyses were performed in SPSS. Perievent histograms of average licking performance following cue onset were compared between mock and stimulation days using two way repeated measures ANOVA (rmANOVA). They were followed by post hoc Bonferroni corrected paired Student's $t$ test analysis of the 5-s histogram bins containing anticipatory behavior $(0-5 \mathrm{~s})$ as well as consummatory behavior (5-10 s). For the locomotion experiment, distance moved over time was compared between mock and stimulation days, using rmANOVA. For assessing performance on the Pavlovian task over trials, trials were split to first 20 and last 20 trials for mock and stim days. In order to analyze decreased performance over trials or days, the licks within the first $15 \mathrm{~s}$ after cue onset were used as a single parameter to assess task performance, and compared over time. To assess the amount of completed trials, the number of trials were counted were animals performed at least one lick within the first $15 \mathrm{~s}$ after cue onset. For the first day after stimulations, only sessions were used when animals were ran on the task $24 \mathrm{~h}$ after initial stimulation. For performance one week later, a session one week after stimulation was picked, where animals received mock stimulation and did not receive any other manipulation. Latency to lick was compared in a similar fashion over days. In all cases rmANOVA were first performed, and followed by paired Student's $t$ tests. 

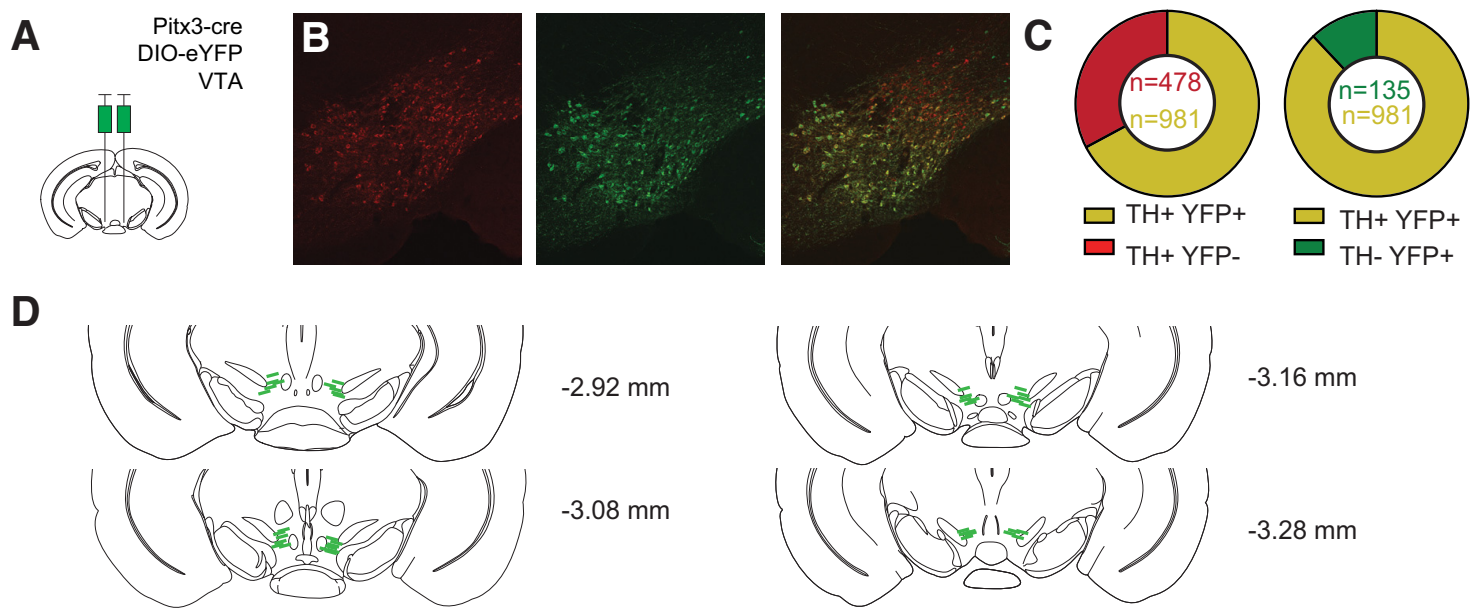

Figure 1. Expression of viral construct in VTA DA neurons of Pitx3-cre mice. A, Schematic illustration of injections. B, Expression of CRE-dependent viral eYFP and TH in the VTA of a Pitx3CRE mouse. Left panel, TH immunoreactivity. Middle panel, eYFP expression. Right panel, Overlap. C, Quantification of eYFP expression among TH positive neurons (left). Quantification of TH expression among eYFP positive neurons (right). $\boldsymbol{D}$, Location of the end-point of the fibers of all animals used in the study.

A

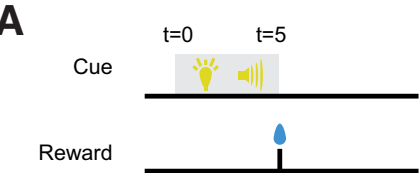

C
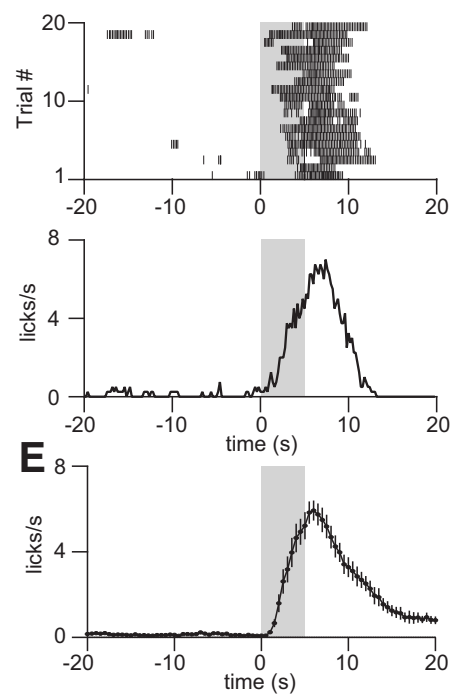

B

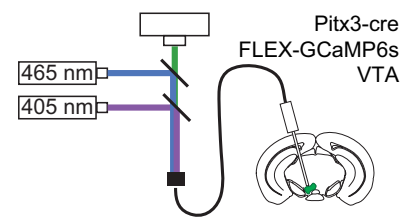

D
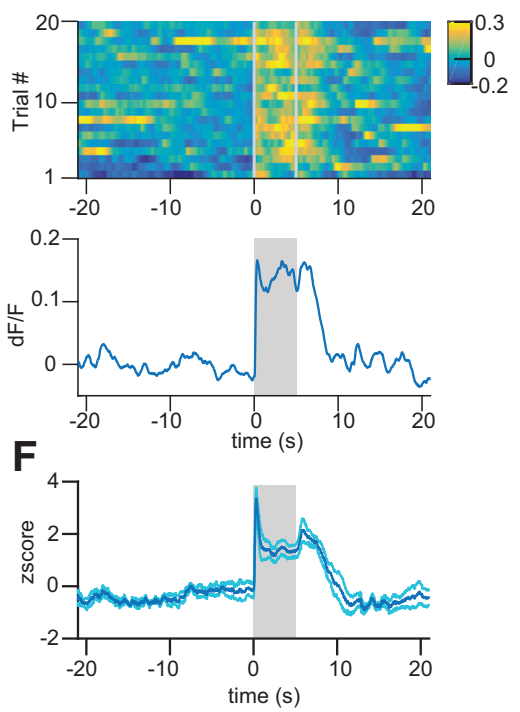

Figure 2. DA activity during Pavlovian conditioning task. $A$, Schematic illustration of Pavlovian conditioning task design. A 5-s cue directly predicts the delivery of a sucrose reward. $\boldsymbol{B}$, Schematic illustration of fiber photometry setup. $\boldsymbol{C}$, Licking behavior around cue onset $(t=0)$ from a single animal and single session (top, trial-by-trial; bottom, average response). Vertical ticks indicate individual licks. D, Fiber photometry VTA DA GCaMP6s signal from same animal as C (top, trial-bytrial; bottom, average response). $\boldsymbol{E}$, Average behavioral licking response of cohort $(n=8)$ of animals trained on Pavlovian conditioning task. $\boldsymbol{F}$, Average fiber photometry signal (dark blue) from animals $(n=5)$ trained on Pavlovian conditioning task. Light blue lines indicate S.E.M.

\section{Results}

Viral targeting of VTA DA neurons in Pitx3-Cre mice and DA calcium dynamics during Pavlovian conditioning task In order to specifically target VTA DA neurons we locally injected CRE-dependent viral constructs into the VTA of Pitx3Cre heterozygote transgenic animals (Smidt et al., 1997, 2012). In order to quantify the efficacy and specificity of this approach we initially injected three animals with a CRE-dependent enhanced YFP,

and waited for four weeks (Fig. 1A). Afterwards, we perfused the animals and performed immunohistochemistry staining for tyrosine hydroxylase $(\mathrm{TH})$. There was clear overlap between virally mediated YFP expression and $\mathrm{TH}$ immunostaining in the VTA (Fig. $1 B$ ). We quantified the amount of overlap in the regions of the VTA that we target throughout this study and found that $\sim 67 \%$ of VTA $\mathrm{TH}+$ neurons also expressed YFP. Moreover, the YFP expression was largely specific to DA neurons as $88 \%$ of the YFP expressing neurons also expressed TH (Fig. 1C). For all the following experiments in this study we also determined the end-location within the VTA of the implanted fibers (Fig. 1D). Animals that did not have fibers in the VTA or did not have any viral expression were excluded from the analysis.

To investigate the role of DA during Pavlovian conditioned behavior we trained freely moving animals on a Pavlovian conditioning task where the presentation of a 5-s audiovisual cue predicts the delivery of a sucrose reward (Fig. 2A). Animals were food restricted to $90 \%$ of their free-feeding weight starting from $2 \mathrm{~d}$ before training, and remained food restricted throughout the experiments. In order to confirm transient DA activity in this task, we injected a second batch of animals with an AAV containing a CRE-dependent GCaMP6s in the VTA, and placed an optic fiber above the area to perform fiber photometry recordings (Fig. 2B). After training animals show robust and consistent licking responses to the audiovisual cue, and also show clear fluorescence signal increases at the moment of cue presentation and the moment of reward delivery (Fig. 2C-F). However, while cues and reward illicit both DA transients and licking behavior, the response profile has a different temporal dynamic. 
A

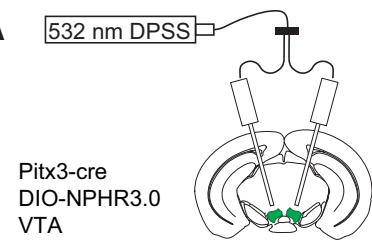

D
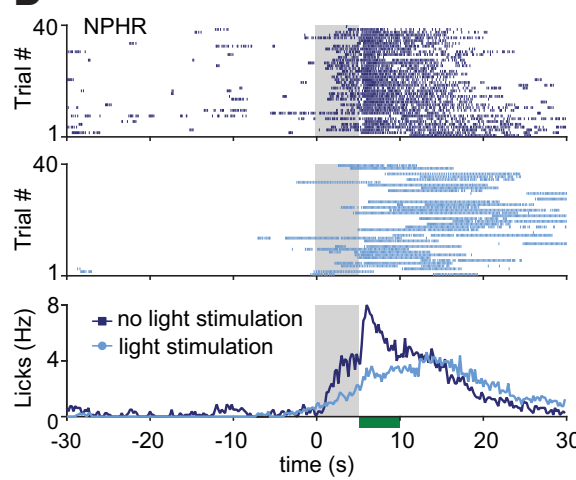

F
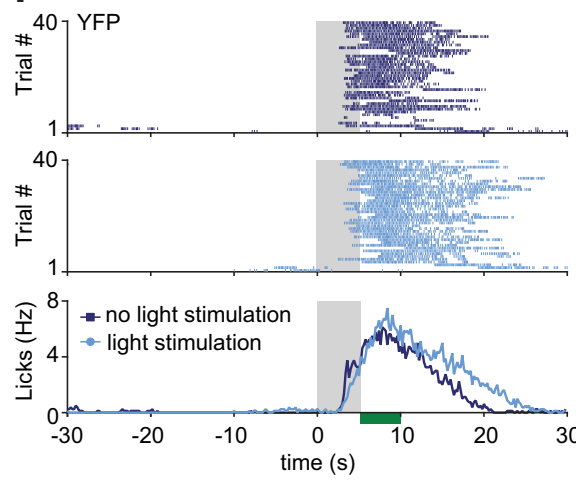

B

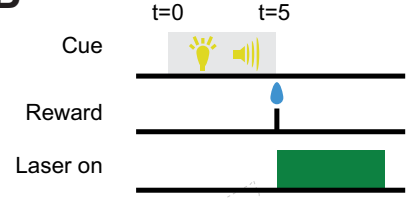

C

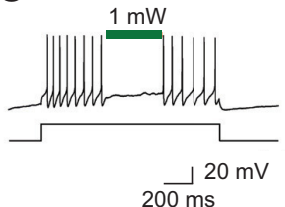

E
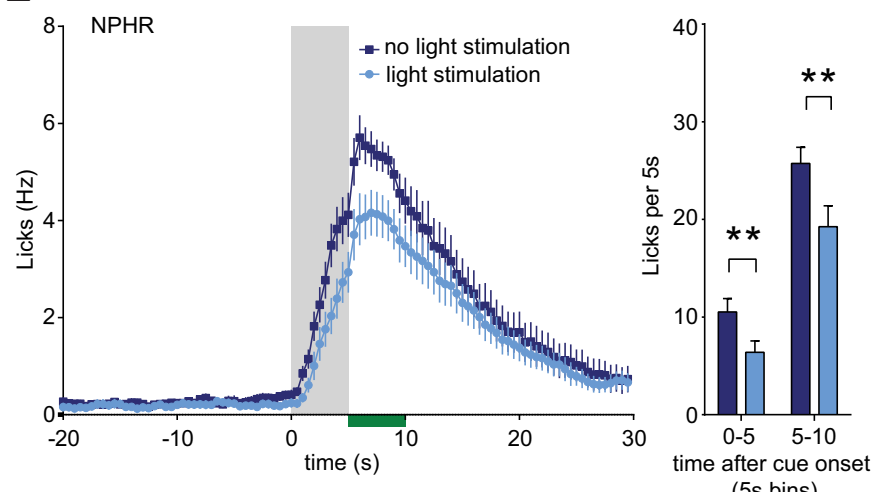

G
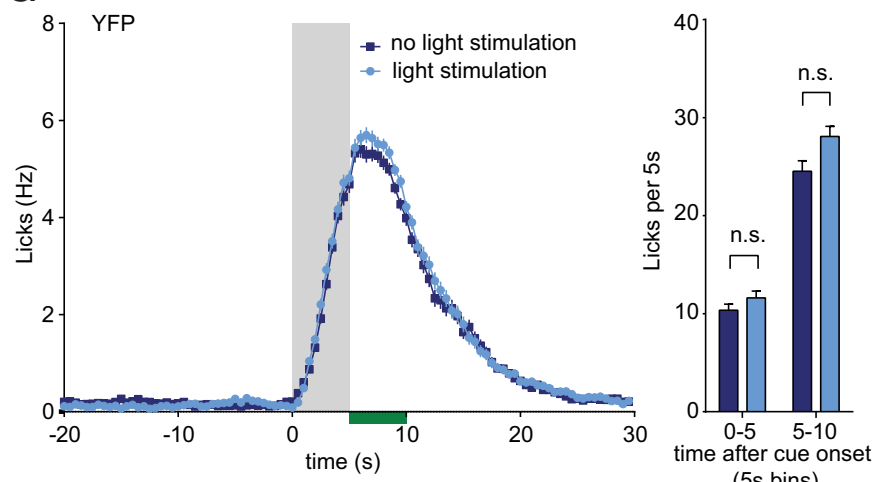

Figure 3. Transient inhibition of VTA DA neurons after reward delivery directly impairs learned behavior for a prolonged period. $\boldsymbol{A}$, Schematic illustration of surgical preparation. $\boldsymbol{B}$, Schematic illustration of DA inhibition during reward delivery ( $t=5-10 \mathrm{~s}$ after cue onset). C, Functional validation of AAV-DI0-NpHR. Whole-cell current clamp recording from AAV-DI0-NpHReYFP expressing VTA DA neurons in brain slices shows that green light $(532 \mathrm{~nm}, 500 \mathrm{~ms}, 1 \mathrm{~mW})$ inhibits action potentials. $\boldsymbol{D}$, Example trial-by-trial and average licking response of a single NpHR-infected animal showing light stimulated and no light stimulated performance. $\boldsymbol{E}$, Average lick rate around cue presentation (left), on mock and light stimulation sessions ( $N=14$ animals for both conditions). Summed 5 -s licking data (right) for periods covering cue presentation $(t=0-5 \mathrm{~s})$ and initial reward consumption $(t=5-10)$. $\boldsymbol{F}$, Example trial-by-trial and average licking response of a single YFP-infected animal showing light stimulated and no light stimulated performance. $\mathbf{G}$, Average lick rate around cue presentation (left), on mock and light stimulation sessions ( $N=11$ animals for both conditions). Summed 5-s licking data (right) for periods covering cue presentation $(t=0-5 \mathrm{~s})$ and initial reward consumption ( $t=5-10)$. In all panels $t=0$ signifies the start of cue presentation. Error bars represent S.E.M. ${ }^{*} p<0.05,{ }^{* *} p<0.01$. n.s. indicates non-significant.

\section{Transient inhibition of VTA DA neurons after reward delivery directly impairs learned behavior for a prolonged period}

We then asked whether inhibition of VTA DA neurons during the reward period would interfere with behavioral licking performance on this Pavlovian conditioning task (Fig. 3A). To investigate this, we injected an AAV either containing a CRE-dependent enhanced halorhodopsin 3.0 (NPHR) or a CRE-dependent YFP in the VTA of Pitx3-Cre heterozygote transgenic animals. Ex vivo slice electrophysiology confirmed that delivery of $532 \mathrm{~nm}$ wavelength laser light inhibited activity of NPHRexpressing neurons (Fig. $3 \mathrm{C}$ ). In trained animals, inhibition of VTA DA neurons during reward delivery in NPHR-expressing animals decreases licking performance $\left(F_{(1,26)}=6.349, p=0.018\right.$, rmANOVA; Fig. $3 D, E)$, during both anticipatory $(T=3.304$, $p=0.011$, paired Student's $t$ test; Fig. $3 E$ ) as well as consummatory phases $(T=3.972, p=0.0032$; Fig. $3 E)$. YFP expressing control animals were unaffected $\left(F_{(1,8)}=1.751, p=0.222,0-5 \mathrm{~s}\right.$ after cue onset: $T=-0.861, p=0.438,5-10 \mathrm{~s}$ after cue onset: $T=$
$-1.448, p=0.221$; Fig. $3 F, G)$. In NPHR-expressing animals' anticipatory behavior was affected following inhibition during reward delivery, indicating that there were prolonged effects on task performance.

In order to investigate the progression of this effect, we created pseudocolored histograms of the average licking response over individual trials. On days without DA inhibition performance is relatively stable over trials, but as DA neurons were inhibited at the moment of reward delivery performance seemed to decrease over trials (Fig. 4A), as was further evident when we quantified the amount of licks in the first $15 \mathrm{~s}$ after cue onset over blocks of trials (time effect $\left.F_{(7,182)}=2.339 p=0.026\right)$, last five trials: $t=2.846, p=$ 0.0127 ; Fig. $4 B$ ). Because animals show this decrease in performance, we also quantified the amount of completed trials. To do this we counted the amount of trials where an animal performs at least one lick in the first $15 \mathrm{~s}$ after cue onset. Without inhibition of DA neurons animals respond on almost every trial, however when DA neurons are 

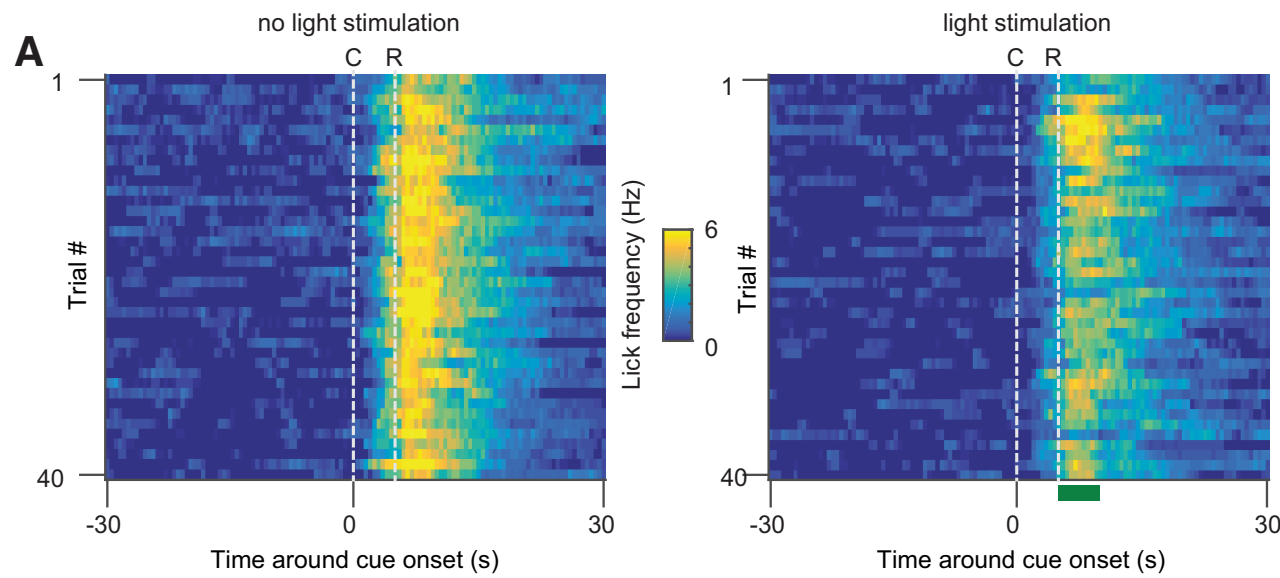

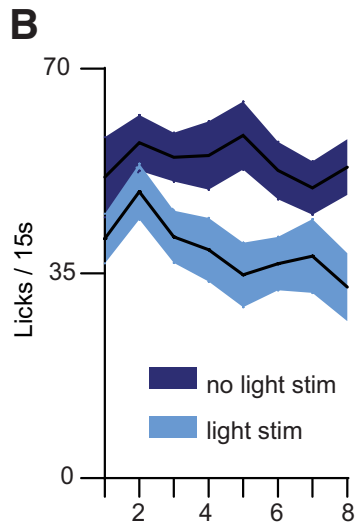

5 trial block average

no light stimulation

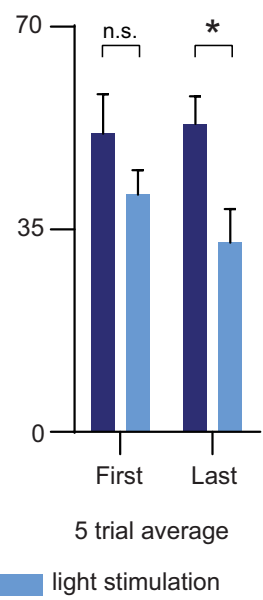

C

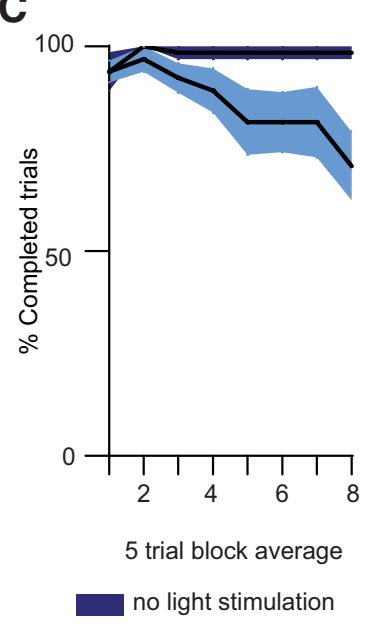

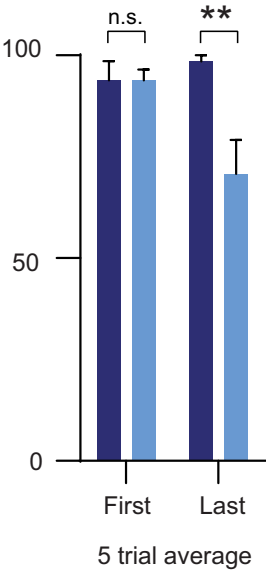

light stimulation
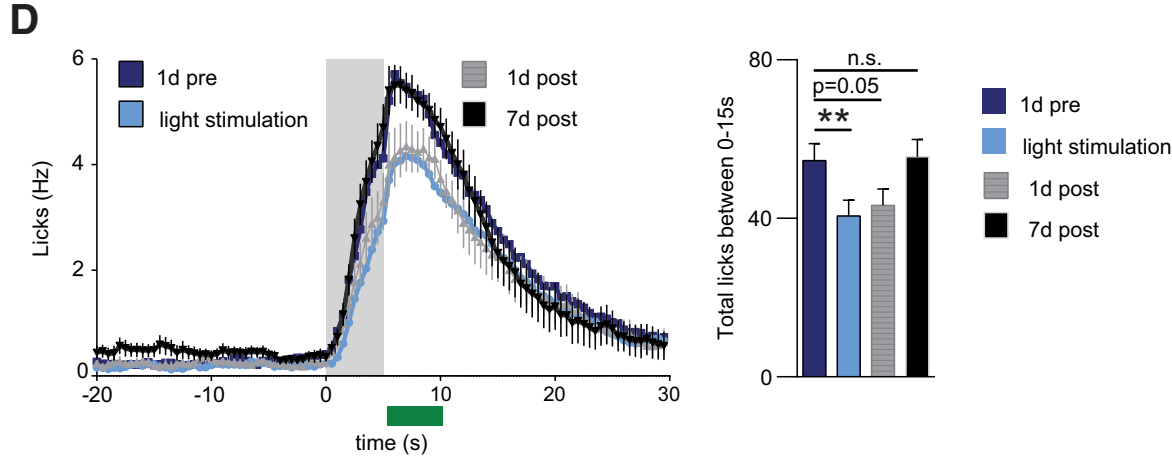

Figure 4. Progressive decrease in performance after inhibition following reward delivery. $A$, Trial-by-trial average performance of all NPHR mice during days of no light stimulation (left panel) or DA inhibition at the reward delivery (right panel). Dotted lines show cue onset (c) and reward delivery (r). $\boldsymbol{B}$, Total licks in the first $15 \mathrm{~s}$ after cue onset during sessions with no light stimulation or light stimulation after reward onset; $* p<0.05, * * p<0.01$. Paired Student's $t$ test. C, Percentage of trials with lick in the first $15 \mathrm{~s}$ after cue onset during session with no light stimulation (dark blue) or light stimulation at reward delivery (light blue). $\boldsymbol{D}$, Average lick rate (left) around cue presentation $(t=0)$, for light stimulation day and no light stimulation day (same as Fig. 3E), as well $1 \mathrm{~d}$ and one week after light stimulation ( $N=14$ for $D A$ inhibition, mock stimulation and week after, $N=13$ for day after). Summed total licks (right) between 0 and $15 \mathrm{~s}$ after cue onset. In all panels, $t=0$ signifies the start of cue presentation. Error bars represent S.E.M. n.s. indicates non-significant.

inhibited during the reward this performance is initially similar, but drops over trials (time effect $F_{(7,182)}=2.616$ $p=0.014$, last five trials: $t=3.791, p=0.0008$; Fig. $4 C$ ). Because of the progressive nature of these effects we then hypothesized that performance on the subsequent day would be affected, in accordance with what others have observed under extinction conditions (Chang et al., 2016). We found that performance on the day following VTA DA inhibition was still decreased, but after repeated training recovered one week after stimulation $(F=6.056, p=0.014,1$ $\mathrm{d}$ before vs stim: $t=3.97, p=0.0008,1 \mathrm{~d}$ before vs $1 \mathrm{~d}$ after: $t=2.15, p=0.026,1 \mathrm{~d}$ before vs $\pm 7 \mathrm{~d}$ after: $t=-0.329$, $p=0.374$; Fig. $4 D$ ). Thus, inhibition of VTA DA neurons during reward consumption progressively weakened anticipatory and consummatory licking over trials and affected the vigor of conditioned and consummatory responses, even on the following day.

\section{Transient inhibition of VTA DA neurons does not impede} licking or locomotion

While VTA DA activity has been associated with reward-predicting cues, DA also regulates motor performance. Furthermore, 
longer inhibition of VTA DA neurons is able to induce aversive responses (Tan et al., 2012). Therefore, we established whether the effects that we observe could be explained by an impediment of licking or locomotion performance. To do this, a different batch of animals were trained to consume (non-predicted) sucrose ad libitum, and we assessed the effects of inhibiting VTA DA neurons during this sucrose consumption. Specifically, we defined onset of a consummatory bout as three licks within half a second, and used that to trigger a 5-s inhibition of VTA DA neurons (Fig. 5A). By introducing a minimal inter stimulation interval, the frequency of stimulation was comparable to that of the earlier Pavlovian conditioning task (for details, see Materials and Methods). We observed no effects of DA inhibition on ongoing sucrose consumption $\left(F_{(1,10)}=0.73, p=0.79\right.$; Fig. $5 B, C)$, and also saw no effects during the $5 \mathrm{~s}$ of light stimulation $(T=$ $-0.663, p=0.54$; Fig. $5 D$ ), or on total licks within the session $(T=-1.269$, $p=0.26$; Fig. $5 E$ ). This was confirmed by a locomotor assay (Fig. $5 F$ ), in which we saw no effects on distance moved during a 5-s inhibition of VTA DA neurons $\left(F_{(1,8)}=0.003, p=0.96\right.$; Fig. $\left.5 G\right)$. Thus, we show that a 5-s inhibition of VTA DA neurons during licking behavior does not impede licking or locomotor performance per se. As a positive control, in these same animals, we tested whether an extended VTA inhibition would be able to induce a place aversion (Fig. $5 H$ ). In this setting, inhibition of VTA DA neurons was able to illicit a place aversion in comparison to YFP expressing control animals $\left(F_{(1,9)}=10.157\right.$, $p=0.011$, day $2: T=3.366, p=0.025$, day 3 : $T=2.583, p=0.089$, day $4: t=2.956, p=$ 0.048 for stimulations days $2-4$, respectively; Fig. 5I). In conclusion, short 5-s inhibition of VTA DA did not alter licking behavior or locomotion, while long-term coupling of inhibition to an environment induces a place avoidance, in line with earlier reports (Tan et al., 2012).

\section{Transient inhibition of VTA DA} neurons during reward-predicting cues directly impairs learned behavior

It was recently shown that inhibition of VTA DA signals following reward delivery, but not during cue presentation, were able to affect task performance (Lee et al., 2020). However, we wondered whether this would also apply to freely moving animals, and while inhibiting throughout the whole behavioral session. We thus set out to inhibit VTA DA
A

B
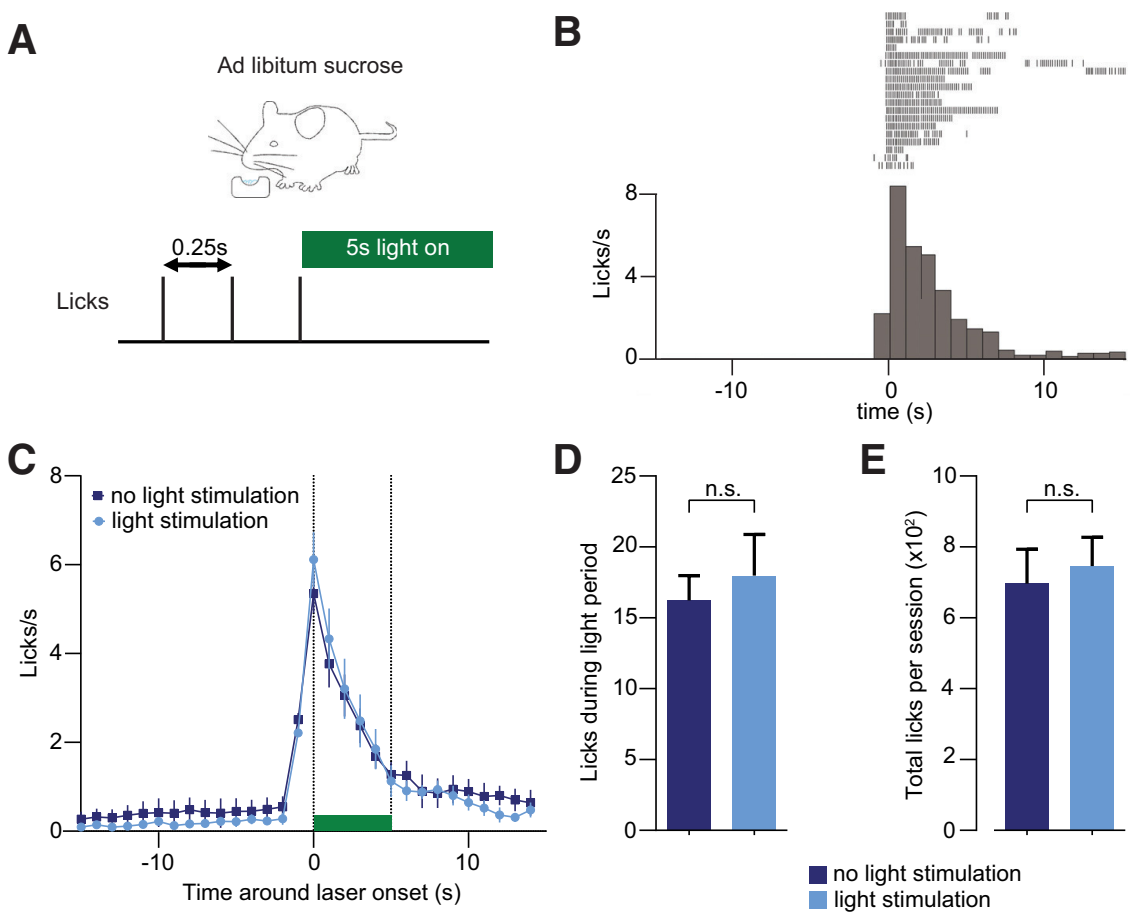

E

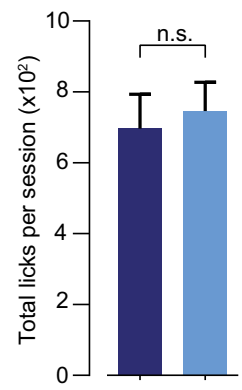

$\mathbf{F}$

G

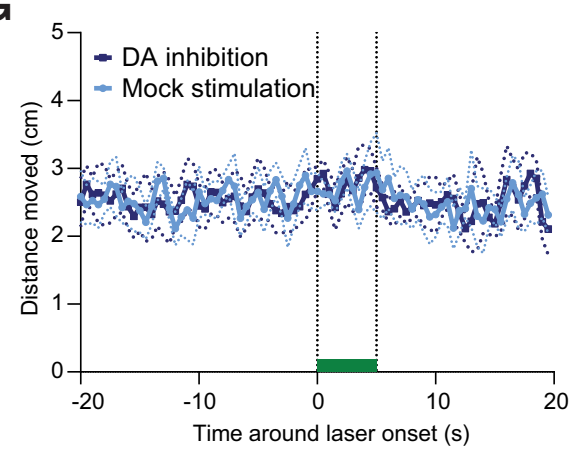

H

\section{I}

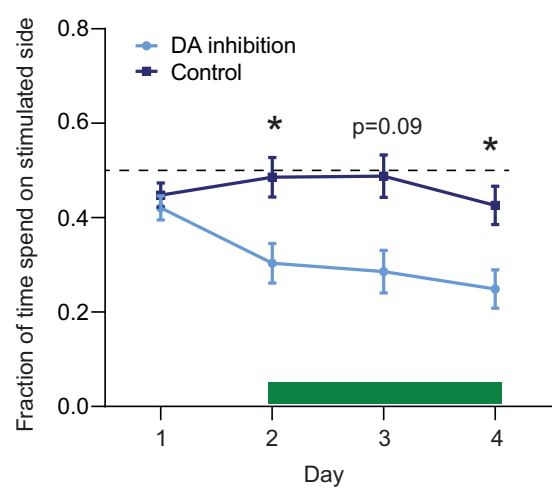

Figure 5. Transient inhibition of VTA DA neurons does not impede licking or cause locomotion deficits. $A$, Schematic illustration of task. Animals had ad libitum access to sucrose, after three licks within $0.5 \mathrm{~s}$, DA neurons were inhibited for $5 \mathrm{~s}$. B, Licking behavior of a single animal around the moment of light stimulation trigger $(t=0)$ during a non-stimulation day. $C$, Average lick rate around stimulation ( $t=0$, green bar), showing inhibition of VTA DA neurons during licking behavior, compared with session with mock optical stimulation ( $N=6$ animals for both conditions). $\boldsymbol{D}$, Average data from $\mathbf{C}$ broken into 5 -s bin during laser stimulation (light blue) or control no light stimulation (dark blue). $\boldsymbol{E}$, Total licks per 30-min session, before and during light stimulation. $\boldsymbol{F}$, Schematic illustration of task. Animals were allowed to freely moving in an open field and unannounced light stimulation occurred for $5 \mathrm{~s}$ every $90 \mathrm{~s}$. G, Average distance moved over time around stimulation ( $t=0$, green bar), comparing between days of stimulation (dark blue) to control days (light blue; $N=5$ animals for both conditions). $\boldsymbol{H}$, Schematic illustration of operant place preference task design. $I$, left, Average fraction of time spend in the stimulated compartment on the first $4 \mathrm{~d}$ of place preference experiment. Animals expressing NPHR in VTA DA neurons are compared with YFP expressing control animals ( $n=4$ YFP animals and $n=7$ NPHR animals). All data are represented as mean $\pm \mathrm{SEM} ; * p<0.05$. n.s. indicates non-significant. 
A

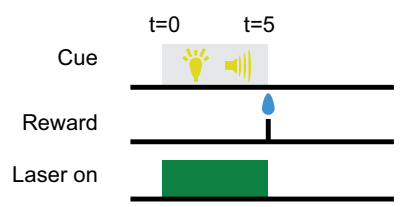

B
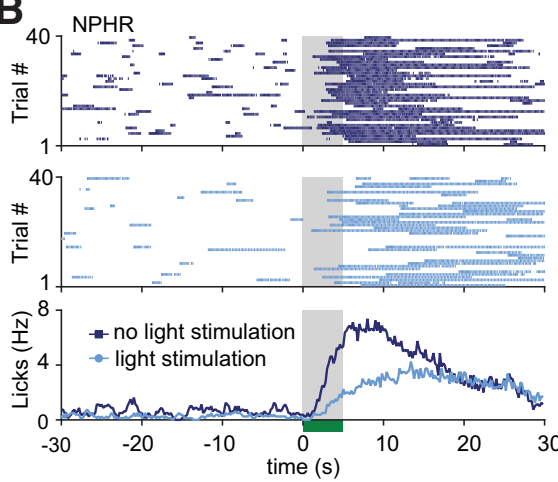

D

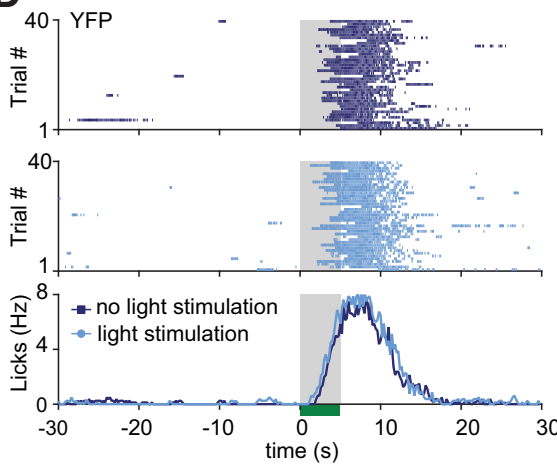

C

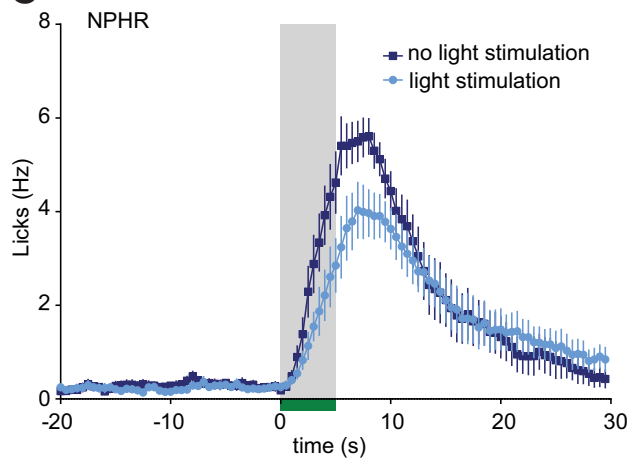

$E$

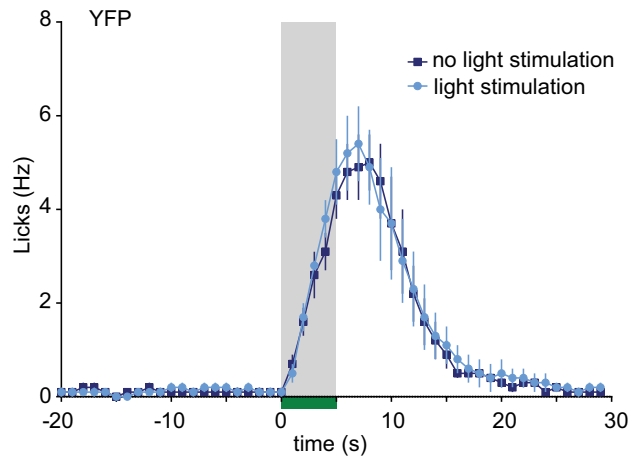

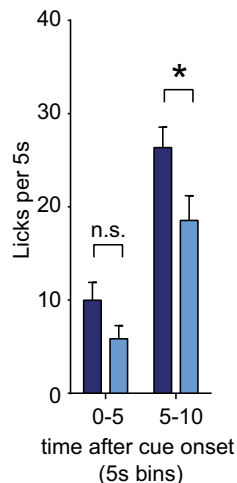

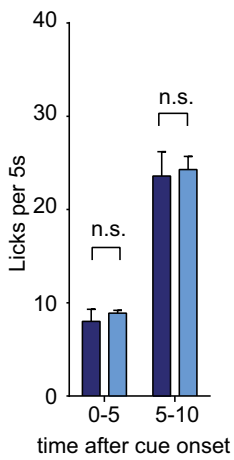

(5s bins)

Figure 6. Transient inhibition of VTA DA neurons during reward-predicting cues directly impairs learned behavior. $A$, Schematic illustration of DA inhibition during cue delivery. $\boldsymbol{B}$, Example trial-by-trial and average licking response of a single NpHR-infected animal showing light stimulated and no light stimulated performance. C, Average lick rate around cue presentation (left), on mock and light stimulation sessions ( $N=9$ animals for both conditions). Summed 5 -s licking data (right) for periods covering cue presentation ( $t=0-5 \mathrm{~s})$ and initial reward consumption $(t=5-10)$. D, Example trial-by-trial and average licking response of a single YFP-infected animal showing light stimulated and no light stimulated performance. $\boldsymbol{E}$, Average lick rate around cue presentation (left), on mock and light stimulation sessions ( $N=5$ animals for both conditions). Summed 5 -s licking data (right) for periods covering cue presentation ( $t=0-5 \mathrm{~s}$ ) and initial reward consumption $(t=5-10)$. Error bars represent S.E.M. ${ }^{*} p<0.05$. n.s. indicates non-significant.

neurons during the 5-s cue presentation in trained animals (Fig. $6 A$ ). Inhibition of VTA DA neurons during the reward-predicting cue decreases licking rate in NPHR-expressing animals $\left(F_{(1,16)}=4.542, p=0.049\right.$; Fig. $\left.6 B, C\right)$. While anticipatory licking during cue presentation was not significantly decreased $(T=$ 2.248, $p=0.11$; Fig. $6 C$ ), licking performance during reward delivery was significantly decreased $(T=3.095, p=0.0296$; Fig. $4 C)$. Control YFP-expressing animals showed no effect of laser illumination of the VTA during the cue $\left(F_{(1,8)}=0.152, p=0.707\right.$, $0-5 \mathrm{~s}$ after cue onset: $T=-0.679, p=0.5,5-10 \mathrm{~s}$ after cue onset $T=-0.175, p=0.9$; Fig. $6 D, E)$.

For these experiments we also considered how these effects develop over trials. We plotted histograms of the average trialby-trial performance during the control and stimulation day (Fig. $7 A$ ), and then again quantified the amount of licks in the first $15 \mathrm{~s}$ after cue onset (Fig. $7 B$ ). Again on days of no DA inhibition performance was stable over the session, but when DA neurons were inhibited performance dropped significantly, but did not change dramatically over trials (stimulation effect $F_{(1,16)}=14.06, p=0.0018$, time effect n.s., first five trials $t=2.802$, $p=0.0171$; Fig. $7 B$ ). This is also evident in the amount of completed trials, where in contrast to DA inhibition during reward delivery, there was no significant decrease in performance (Fig. 7C). Thus, we conclude that inhibition of VTA DA neurons during cues predicting food reward directly decreased conditioned responses without showing trial-by-trial worsening of the performance.

\section{Discussion}

VTA DA neurons increase their activity to primary rewards and reward-predictive cues; however, it is unclear to what extent this firing is necessary for expression of learned associative behavior on the short and long-term. Here, we specifically transiently inhibited the activity of these neurons during either cue or reward presentation in animals trained on a Pavlovian conditioning task. In line with earlier work (Steinberg et al., 2013; Chang et al., 2016), when we inhibit during reward delivery, mimicking a negative RPE, we observe chronic decreases that develops over trials and persist onto the next day. In contrast, inhibition of cue-induced DA activity directly and stably decreases conditioned and consummatory licking performance. Together, 
A

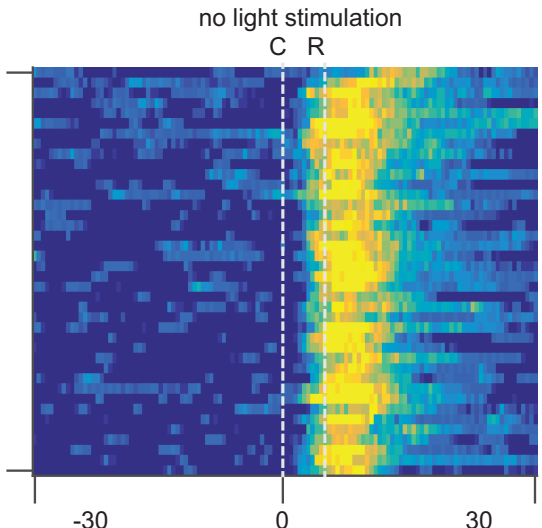

Time around cue onset (s)

B

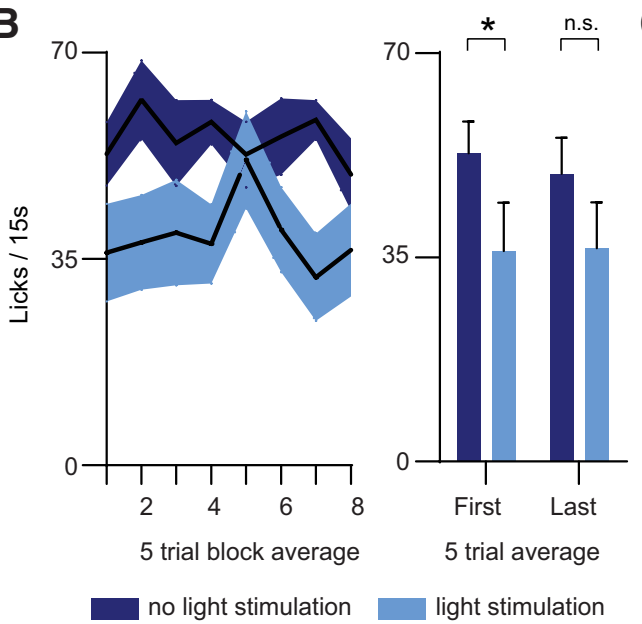

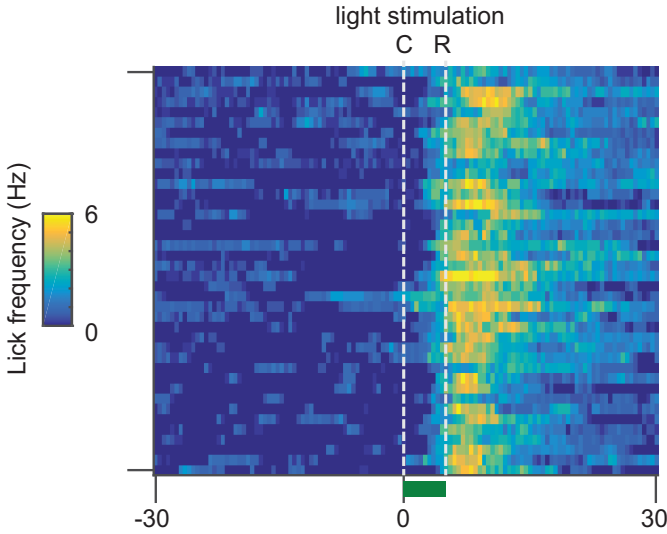

Time around cue onset (s)
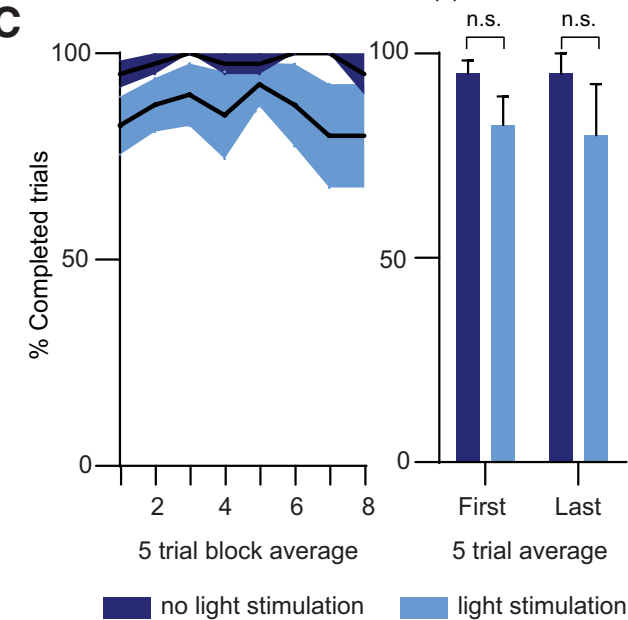

Figure 7. Inhibition of VTA DA during cue presentation does not lead to a progressive loss in performance. $A$, Trial-by-trial average performance over NPHR mice during days of no light stimulation (left panel) or DA inhibition at the reward delivery (right panel). Dotted lines show cue onset (c) and reward delivery ( $r$ ). $\boldsymbol{B}$, Total licks in the first $15 \mathrm{~s}$ after cue onset during sessions with no light stimulation or light stimulation during $(S ; * p<0.05, * * p<0.01$. Paired Student's $t$ test. C, Percentage of trials with lick in the first $15 s$ after cue onset during session with no light stimulation (dark blue) or light stimulation during CS (light blue). Error bars represent S.E.M. n.s. indicates non-significant.

these data suggest that phasic VTA DA activity during both predictive cues and reward delivery are both necessary for behavioral performance, yet play different roles in maintaining trained behavior.

Reward-predictive cues elicit behavioral approach and responding, in our assay this takes the form of anticipatory licking behavior. This phenomena has been explained through different theoretical frameworks, where reward-predicting cues encode a RPE (Schultz et al., 1997), gain incentive salience (Berridge, 2007), elicit behavioral activation (Robbins and Everitt, 1992, 2007), or elicit flexible approach (Nicola, 2010). Others have suggested DA signals encode a temporal discounted future reward value (Hamid et al., 2016) driving appetitive behavior. While our data do not rule out any aforementioned theory, it suggests that cue-related and reward-related phasic DA activity is directly necessary for appetitive behavior, as we observed a direct within-session decrement in licking performance. Moreover, the more chronic effects that we observe, highlight an established role for phasic DA signaling in reinforcement learning.

One of the crucial questions for these theories on DA function is whether VTA DA activity, following cues or rewards, is directly involved in reward-seeking behavior. Following a classical RPE valuation by VTA DA, activity of these neurons might be important for learning aspects, but not necessarily affect direct trial-by-trial behavior. Manipulations of VTA DA activity through electrical stimulation, 6-OHDA lesions or pharmacological receptor blockade have suggested that indeed VTA DA is directly linked to, and necessary for, reward-related response behavior (Parkinson et al., 2002; du Hoffmann and Nicola, 2014). However, these studies lack the temporal resolution to specifically manipulate VTA DA activity during stimuli presentation only. Optogenetic-based studies have shown that manipulations of VTA DA neurons can alter associative learning, as activating VTA DA neurons during a blocked cue, increases future approach to that cue (Steinberg et al., 2013), and decreasing VTA DA activity induces a down-valuation of future cueinduced responses (Chang et al., 2016). While these studies implicate DA in reward-learning processes on subsequent days, in the current study we show that inhibition of VTA DA neurons during reward-predicting cues can directly decrease conditioned and consummatory behavior during reward seeking. This is in line with reports that activation of VTA DA neurons directly reinforces chosen action on a subsequent trial (Hamid et al., 2016), and is suggested by VTA DA reinforcement studies (Witten et al., 2011; Kim et al., 2012; Ilango et al., 2014).

In this study, we chose to employ a task design that was a minimalistic Pavlovian conditioning task, because of the extensive history of looking at DA activity in similar setups. Because of the freely-moving nature of the task and the Pavlovian design, 
we chose to use cues that were purposefully not restricted to a particular location within the chamber. We reasoned that this would lead to more robust behavior, as animals would experience the cues roughly in the same way regardless of their location within the experimental cage. However, the downside is that this makes it difficult to differentiate between specific behavioral strategies that animals might choose to employ, like goal or signtracking. Specifically the performance sign-tracking animals, that develop clear cue-induced DA transients, is dependent on DA during Pavlovian conditional approach behavior (Flagel et al., 2011). Future studies might elucidate how these short timelocked optogenetic inhibitions differentially effect sign versus goal-tracking behaviors.

It has recently been shown that DA inhibition directly affects learned associative performance in head fixed mice (Lee et al., 2020). While this study primarily replicates some of our findings, there are some striking differences. While their reward-coupled inhibition on a subset of trials only transiently decreases performance, we find that after our whole-session inhibition, performance is still diminished on the following day. This is in line with the consensus in the field that these reward-related responses strongly impact future learning (Steinberg et al., 2013; Chang et al., 2016). Moreover, we find that inhibitions during the cue period drastically decreases licking performance, Lee et al. (2020) find no effect of cue-coupled inhibition. Possibly these differences arise from the freely moving versus head fixed nature of the different designs. While we only measure licking behavior, the nature of our naturalistic freely moving settings means cue presentation typically also requires that animals approach the reward receptacle, requiring approach behavior that has been shown to be dependent on VTA DA activation (Saunders et al., 2018). Thus, in our experiments, it is hard to differentiate between effects on cue-induced or approach-related transient DA activity as they likely occur in a relatively short time-period after cue onset, and effects on either of them likely result in decreased licking behavior.

Perhaps this combination of cue-elicited and approach-elicited DA could help explain the difference between the effects we observe over trials between cue and reward-related DA inhibitions. While the progressive effects of reward delivery coupled DA inhibition largely follows what one would expect during reward omissions (where DA neurons show decreases in activity), the effects of cue-coupled DA inhibition are not clearly changing over trials. As we suggest above, this could be attributed to the role DA plays in approach behavior (Saunders et al., 2018). In contrast, it could also be that this DA decreases the relative desirability of the cue, causing a decrease in performance. In task designs where the animal has multiple options between rewards, DA signals at the cue relate to the preferred reward (Roesch et al., 2007). Both these options could directly change the intensity of the animals' subsequent licking behavior and it would be worthwhile to explore this further using task designs that are better optimized to separate these DA signals.

While we are able to repeat earlier observations that prolonged DA inhibition induces a place aversion (Tan et al., 2012), in the current study, inhibition of VTA DA neurons during active licking behavior does not decrease licking per se. This indicates that while longer DA inhibition might directly initiate aversive responses, the short inhibition that we use in this assay is not sufficient to induce an aversive state that is sufficient to stop licking behavior. Moreover, this also suggests that the effects we see are not mediated by motoric impairments because of inhibition of VTA DA neurons. These results are confirmed by our finding that a brief optogenetic inhibition does not alter locomotion. Therefore, we consider that the effects are likely mediated by a direct decrease of VTA DA phasic activity during rewards and reward-predicting cues, and suggest that decreasing this signaling impairs reward-seeking behaviors.

During omission trials in conditioning tasks, in which rewards are unexpectedly not delivered, activity of VTA DA neurons decreases below baseline levels (Schultz et al., 1997; Cohen et al., 2012), encoding a proposed negative RPE. While our optogenetic inhibition of VTA DA neurons during reward presentation might mimic such a signal, it should be considered that the reward is still delivered, and animals typically still consume all of the reward. Thus, it is likely that our manipulation does not completely mimic the endogenous brain activity that occurs during these reward omissions, giving us a unique opportunity to assess the effects of negative RPE DA signaling without changes in expectation because of reward unavailability. We find that licking performance directly decreases, and decreases over trials, while remaining lowered on the day after DA inhibition. Thus, we consider that the inhibition of VTA DA neurons during reward delivery decreases the reinforcing properties of the reward, resulting in prolonged impairments in performance.

Interestingly, earlier work using halorhodopsin mediated inhibition in TH-cre rats was unable to observe direct effects of inhibition on task performance (Chang et al., 2016). However, we set out our task design such that animals show large, yet consistent, amounts of anticipatory and consummatory task-related performance. This allowed us to optimally quantify smaller deviations in performance. It should be considered that although behavior is partially impaired, performance is not completely abolished. In fact, we find that toward the end of the session, all sucrose is typically consumed. While the parsimonious explanation would be that we were not able to fully silence all VTA DA activity, it is interesting to consider that this behavior remains partially intact while VTA DA neurons are inhibited.

We find that VTA DA inhibition only impairs licking behavior in a context where animals associate this behavior with the presence of a temporally discrete environmental cue. It is interesting to consider these findings in the light of cue induced feeding literature, although in such studies animals are specifically kept on ad libitum food to minimize the influence of hungerdriven food seeking (Petrovich, 2013). Nevertheless, many of the involved brain structures (e.g., lateral hypothalamus, amygdala and prefrontal cortex), and neuromodulators (e.g., orexin, ghrelin) are closely interconnected with VTA DA neurons (Meye and Adan, 2014). As such, VTA DA neurons might play an important role in the overconsumption of food in obesogenic environments (Berthoud, 2012).

Overall, this study shows that inhibition of VTA activity following rewards and reward-predictive cues directly impairs performance. Moreover, performance is decreased over time and remains decreased the day after stimulation, suggesting impairments in reinforcing capacity. These data support earlier reports on VTA DA involvement in associative learning, but also provide compelling evidence for a direct role of phasic VTA DA activity in reinforcement and cue-induced appetitive responses.

\section{References}

Adamantidis AR, Tsai HC, Boutrel B, Zhang F, Stuber GD, Budygin EA, Tourino C, Bonci A, Deisseroth K, de Lecea L (2011) Optogenetic interrogation of dopaminergic modulation of the multiple phases of rewardseeking behavior. J Neurosci 31:10829-10835. 
Berridge KC (2007) The debate over dopamine's role in reward: the case for incentive salience. Psychopharmacology 191:391-431.

Berthoud HR (2012) The neurobiology of food intake in an obesogenic environment. Proc Nutr Soc 71:478-487.

Brown MTC, Tan KR, O'Connor EC, Nikonenko I, Muller D, Lüscher C (2012) Ventral tegmental area GABA projections pause accumbal cholinergic interneurons to enhance associative learning. Nature 492:452-456.

Castellanos FX, Tannock R (2002) Neuroscience of attention-deficit/hyperactivity disorder: the search for endophenotypes. Nat Rev Neurosci 3:617-628.

Chang CY, Esber GR, Marrero-Garcia Y, Yau HJ, Bonci A, Schoenbaum G (2016) Brief optogenetic inhibition of dopamine neurons mimics endogenous negative reward prediction errors. Nat Neurosci 19:111-116.

Cohen JY, Haesler S, Vong L, Lowell BB, Uchida N (2012) Neuron-type-specific signals for reward and punishment in the ventral tegmental area. Nature 482:85-88.

Day JJ, Roitman MF, Wightman RM, Carelli RM (2007) Associative learning mediates dynamic shifts in dopamine signaling in the nucleus accumbens. Nat Neurosci 10:1020-1028.

Di Ciano P, Cardinal RN, Cowell RA, Little SJ, Everitt BJ (2001) Differential involvement of NMDA, AMPA/kainate, and dopamine receptors in the nucleus accumbens core in the acquisition and performance of Pavlovian approach behavior. J Neurosci 21:9471-9477.

du Hoffmann J, Nicola SM (2014) Dopamine invigorates reward seeking by promoting cue-evoked excitation in the nucleus accumbens. J Neurosci, 34:14349-14364.

Fields HL, Hjelmstad GO, Margolis EB, Nicola SM (2007) Ventral tegmental area neurons in learned appetitive behavior and positive reinforcement. Annu Rev Neurosci 30:289-316.

Flagel SB, Clark JJ, Robinson TE, Mayo L, Czuj A, Willuhn I, Akers CA, Clinton SM, Phillips PEM, Akil H (2011) A selective role for dopamine in stimulus-reward learning. Nature 469:53-57.

Hamid AA, Pettibone JR, Mabrouk OS, Hetrick VL, Schmidt R, Vander Weele CM, Kennedy RT, Aragona BJ, Berke JD (2016) Mesolimbic dopamine signals the value of work. Nat Neurosci 19:117-126.

Howes OD, Kapur S (2009) The dopamine hypothesis of schizophrenia: version III - The final common pathway. Schizophr Bull 35:549-562.

Ilango A, Kesner AJ, Keller KL, Stuber GD, Bonci A, Ikemoto S (2014) Similar roles of substantia nigra and ventral tegmental dopamine neurons in reward and aversion. J Neurosci 34:817-822.

Kim KM, Baratta MV, Yang A, Lee D, Boyden ES, Fiorillo CD (2012) Optogenetic mimicry of the transient activation of dopamine neurons by natural reward is sufficient for operant reinforcement. PLoS One 7: e33612.

Lee K, Claar LD, Hachisuka A, Bakhurin KI, Nguye J, Trott JM, Gill JL, Masmanidis SC (2020) Temporally restricted dopaminergic control of reward-conditioned movements. Nat Neurosci 23:209-216.

Lerner TN, Shilyansky C, Davidson TJ, Evans KE, Beier KT, Zalocusky KA, Crow AK, Malenka RC, Luo L, Tomer R, Deisseroth K (2015) Intactbrain analyses reveal distinct information carried by $\mathrm{SNc}$ dopamine subcircuits. Cell 162:635-647.

Meye FJ, Adan RA (2014) Feelings about food: the ventral tegmental area in food reward and emotional eating. Trends Pharmacol Sci 35:31-40.

Nestler EJ, Carlezon WA (2006) The mesolimbic dopamine reward circuit in depression. Biol Psychiatry 59:1151-1159.

Nicola SM (2010) The flexible approach hypothesis: unification of effort and cue-responding hypotheses for the role of nucleus accumbens dopamine in the activation of reward-seeking behavior. J Neurosci 30:16585-16600.

Nicola SM, Taha SA, Kim SW, Fields HL (2005) Nucleus accumbens dopamine release is necessary and sufficient to promote the behavioral response to reward-predictive cues. Neuroscience 135:1025-1033.

Pan WX, Schmidt R, Wickens JR, Hyland BI (2005) Dopamine cells respond to predicted events during classical conditioning: evidence for eligibility traces in the reward-learning network. J Neurosci 25:6235-6242.

Parkinson JA, Dalley JW, Cardinal RN, Bamford A, Fehnert B, Lachenal G, Rudarakanchana N, Halkerston KM, Robbins TW, Everitt BJ (2002) Nucleus accumbens dopamine depletion impairs both acquisition and performance of appetitive Pavlovian approach behaviour: implications for mesoaccumbens dopamine function. Behav Brain Res 137:149-163.
Pascoli V, Terrier J, Hiver A, Lüscher C (2015) Sufficiency of mesolimbic dopamine neuron stimulation for the progression to addiction. Neuron 88:1054-1066.

Petrovich GD (2013) Forebrain networks and the control of feeding by environmental learned cues. Physiol Behav 121:10-18.

Phillips PEM, Stuber GD, Heien MLAV, Wightman RM, Carelli RM (2003) Subsecond dopamine release promotes cocaine seeking. Nature 422:614618.

Robbins TW, Everitt BJ (1992) Functions of dopamine in the dorsal and ventral striatum. Semin Neurosci 4:119-127.

Robbins TW, Everitt BJ (2007) A role for mesencephalic dopamine in activation: commentary on Berridge (2006). Psychopharmacology 191:433437.

Roesch MR, Calu DJ, Schoenbaum G (2007) Dopamine neurons encode the better option in rats deciding between differently delayed or sized rewards. Nat Neurosci 10:1615-1624.

Roitman MF, Stuber GD, Phillips PE, Wightman RM, Carelli RM (2004) Dopamine operates as a subsecond modulator of food seeking. J Neurosci 24:1265-1271.

Root DH, Mejias-Aponte CA, Zhang S, Wang HL, Hoffman AF, Lupica CR, Morales M (2014) Single rodent mesohabenular axons release glutamate and GABA. Nat Neurosci 17:1543-1551.

Salamone JD, Correa M (2012) The mysterious motivational functions of mesolimbic dopamine. Neuron 76:470-485.

Saunders BT, Richard JM, Margolis EB, Janak PH (2018) Dopamine neurons create Pavlovian conditioned stimuli with circuit-defined motivational properties. Nat Neurosci 21:1072.

Schultz W, Dayan P, Montague RP (1997) A neural substrate of prediction and reward. Science 275:1593-1599.

Sharpe MJ, Chang CY, Liu MA, Batchelor HM, Mueller LE, Jones JL, Niv Y, Schoenbaum G (2017) Dopamine transients are sufficient and necessary for acquisition of model-based associations. Nat Neurosci 20:735-742.

Smidt MP, van Schaick HSA, Lanctot C, Tremblay JJ, Cox JJ, van der Kleij AAM, Wolterink G, Drouin J, Burbach JPH (1997) A homeodomain gene Ptx3 has highly restricted brain expression in mesencephalic dopaminergic neurons. Proc Natl Acad Sci USA 94:13305-13310.

Smidt MP, von Oerthel L, Hoekstra EJ, Schellevis RD, Hoekman MFM (2012) Spatial and temporal lineage analysis of a Pitx3-driven cre-recombinase knock-in mouse model. PLoS One 7:e42641.

Sparta DR, Stamatakis AM, Phillips JL, Hovelsø N, van Zessen R, Stuber GD, (2011) Construction of implantable optical fibers for long-term optogenetic manipulation of neural circuits. Nat Protoc 7:12-23.

Steinberg EE, Keiflin R, Boivin JR, Witten IB, Deisseroth K, Janak PH (2013) A causal link between prediction errors, dopamine neurons and learning. Nat Neurosci 16:966-973.

Stuber GD, Hnasko TS, Britt JP, Edwards RH, Bonci A (2010) Dopaminergic terminals in the nucleus accumbens but not the dorsal striatum corelease glutamate. J Neurosci 30:8229-8233.

Tan KR, Yvon C, Turiault M, Mirzabekov JJ, Doehner J, Labouèbe G, Deisseroth K, Tye KM, Lüscher C (2012) GABA neurons of the VTA drive conditioned place aversion. Neuron 73:1173-1183.

Wise R (2004) Dopamine, learning and motivation. Nat Rev Neurosci 5:483494.

Witten IB, Steinberg EE, Lee SY, Davidson TJ, Zalocusky KA, Brodsky M, Yizhar O, Cho SL, Gong S, Ramakrishnan C, Stuber GD, Tye KM, Janak PH, Deisseroth K (2011) Recombinase-driver rat lines: tools, techniques, and optogenetic application to dopamine-mediated reinforcement. Neuron 72:721-733.

Yang H, de Jong JW, Tak Y, Peck J, Bateup HS, Lammel S (2018) Nucleus accumbens subnuclei regulate motivated behavior via direct inhibition and disinhibition of VTA dopamine subpopulations. Neuron 97:434449.e4.

van Zessen R, Phillips JL, Budygin EA, Stuber GD (2012) Activation of VTA GABA neurons disrupts reward consumption. Neuron 73:1184-1194.

Zweifel LS, Parker JG, Lobb CJ, Rainwater A, Wall VZ, Fadok JP, Darvas M, Kim MJ, Mizumori SJY, Paladini CA, Phillips PEM, Palmiter RD (2009) Disruption of NMDAR-dependent burst firing by dopamine neurons provides selective assessment of phasic dopamine-dependent behavior. Proc Natl Acad Sci USA 106:7281-7288. 